\title{
Erratum to: Anisotropic compact sphere with Van der Waals equation of state
}

\author{
S. Thirukkanesh • F.C. Ragel
}

Received: 13 August 2014 / Accepted: 13 August 2014 / Published online: 18 September 2014

(C) Springer Science+Business Media Dordrecht 2014

Erratum to: Astrophys. Space Sci. (2014)

DOI 10.1007/s10509-014-1883-1

After our paper was published in the Online First version, we were alerted by Dr. Manuel Malaver de la Fuente who considered the same type of equation of state to model a quark star (Malaver 2013).

\section{References}

Malaver, M.: World Applied Programming 3, 309 (2013)

The online version of the original article can be found under doi:10.1007/s10509-014-1883-1.

S. Thirukkanesh $(\bowtie)$

Department of Mathematics, Eastern University, Chenkalady,

Sri Lanka

e-mail: thirukkanesh@yahoo.co.uk

F.C. Ragel

Department of Physics, Eastern University, Chenkalady,

Sri Lanka 